\title{
El proyecto de los lugares del trabajo
}

Horacio Torrent Profesor, Escuela de Arquitectura, Pontificia Universidad Católica de Chile

Debido a las nuevas dimensiones que adquiere la secuencia laboral por medio de redes informáticas, los lugares de trabajo mantienen su relevancia y significación en la ciudad contemporánea. La fábrica y la oficina se constituyen en lugares de trabajo emblemáticos que la arquitectura ha enfrentado a través de variadas opciones. A partir de estos referentes, el autor pone en contexto las obras publicadas en este número.

IDEOLOGÍA, TÉCNICA Y PRÁCTICA / Trabajo es por cierto una palabra muy general, que refiere tanto a hacer algo, como a algo hecho, y a muchas de las acciones que ello implica. En la diversidad de significados que la palabra asume cotidianamente, hay que distinguir unos pocos que permiten centrar el discurso.

La noción de trabajo está asociada a un esfuerzo por ganarse la vida; y tal vez, a una ética laboral que organiza la vida de las personas al menos en occidente desde hace, al menos, unos doscientos años ${ }^{1}$. Por una parte, implica una cierta constancia, al mismo tiempo que una cierta regularidad. Por otra, la obtención de un efecto, sea una obra, un producto, o algo más difuso, incluso un reconocimiento.

El uso habitual del término advierte sobre la significación amplia de una ocupación en algo, retribuida de alguna manera. En tiempos de la cultura agrícola, el trabajo significó asegurar la alimentación, la sobrevivencia, por medio de la labor de la tierra ${ }^{2}$. En tiempos más recientes, desde el desarrollo de las relaciones productivas capitalistas, la especialización del término alude a una ocupación regular con una retribución en dinero, sea en las formas del desempeño de un oficio, una profesión o una práctica de manera liberal, así como simplemente un empleo regular y pago. De hecho, el tiempo en que no se trabaja es -en la sociedad actual- habitualmente referido como tiempo libre, es decir, no sujeto de regularidad, procedimientos instituidos, horario, cierto tiempo de duración y concurrencia al lugar donde el trabajo se desarrolla.

Por cierto que el trabajo en su organización moderna ha considerado al lugar como un tópico de importancia. Más recientemente, la tecnología informática, ha motivado intentos de ordenamiento de la secuencia laboral por medio de redes, con la consecuente deslocalización del trabajo. Pero por cierto que, aún cuando crece el trabajo vehiculado por redes, los lugares del trabajo siguen siendo importantes, al menos así lo verifica el aumento sistemático de la superficie de oficinas ante la terciarización de la sociedad y el grado de significación que alcanzan en las ciudades.

Los lugares de trabajo han sido -históricamente- muchos y variados, nadie duda que hay en los más diversos edificios personas trabajando: en las escuelas, en los comercios, en los parlamentos, o en los parques temáticos, entre otros.

Sin embargo, es indudable que desde fines del s. XIX hay dos tipos de edificios que se han llevado la primacía en relación con la significación que el trabajo adquiere en la sociedad: la fábrica y la oficina. Muchos autores los han relacionado con la experiencia de la Arquitectura Moderna. Banham expuso con claridad cómo la fábrica, junto con el elevador de granos, fue uno de los tipos que influyeron radicalmente en la configuración ideológica y estética de la Arquitectura Moderna ${ }^{3}$. Las fábricas de Albert Kahn en Detroit y otras ciudades norteamericanas en torno a los grandes lagos, fueron paradigmas constructivos tanto para los arquitectos de Europa Occidental, como para los soviéticos. Los
Most due to the new dimensions that the labor sequence acquires through information networks, work places maintain their relevance and meaning in the contemporary city. The factory and the office become emblematic work places that architecture has come to grips with through various options. Through these references the author puts the works published in this number in context.

edificios de oficinas de Sullivan o de Burham en Chicago estarían en igual medida fuertemente presentes.

En el deslumbramiento de los arquitectos europeos por la realidad norteamericana, había un contenido conceptual clave: la organización del trabajo. Algo que, por cierto, deslumbraba también a los políticos, a los administradores, y a muchos sectores de la sociedad, desde Lenin hasta Clemenceau, desde Ginzburg a Le Corbusier ${ }^{4}$.

En 1911 Taylor publicó The principles of scientific management, que resumía su experiencia de más de 20 años en la organización de plantas de cortes de metal y exponía muchos de los tópicos que había aplicado sistemáticamente en la organización racional del trabajo ${ }^{5}$. La propuesta de Taylor fue básicamente un método de disciplina del trabajo y de organización de la producción basado en investigaciones científicas sobre la eficiencia laboral por medio de la aplicación de un sistema de pago por rendimiento ${ }^{6}$.

El método de Taylor prácticamente inundó el pensamiento occidental, trasladándose a las más variadas disciplinas e incluso a formas de la organización social. Pero, obviamente, su impacto mayor lo obtuvo a partir de la incorporación de sus ideas y reformulación en la empresa de Henry Ford y la concepción de la línea de montaje, por medio de la organización diferenciada del trabajo.

En arquitectura, indudablemente influiría en la concepción del espacio del trabajo en relación a las rutinas, a las jerarquías y obviamente a la división de funciones, en los edificios. Pero también fueron claramente asumidas por Le Corbusier para su aplicación urbanística en el Plan Voisin y en la propuesta de la Ville Contemporaine ${ }^{7}$. Se refirió, junto con Ozenfant en Acerca del purismo, a las posibilidades que brindaba "el programa riguroso de la fábrica moderna”; y más específicamente al taylorismo, advirtiendo que no se trataba "sino de explotar inteligentemente los descubrimientos científicos. El instinto, el tantear, el empirismo son substituidos por los principios científicos del análisis, por la organización y la clasificación”.

Pero no fue sólo un hálito genérico que sobrevoló las mentes de los arquitectos para adecuar la disciplina a los tiempos, sino que también se procesó y formuló como base del propio trabajo del arquitecto. Las formas de la organización taylorista se transferirían también como un instrumental para la concepción del proyecto de arquitectura y constituirían un paradigma para la práctica y técnica del proyecto funcionalista.

Moisei Ginzburg abogaba en 1926 por un nuevo método de pensamiento arquitectónico, en el que "un problema arquitectónico, como cualquier otro problema, sólo se resuelve mediante la exacta determinación de las incógnitas y la búsqueda del método justo para llegar a la solución”, porque a su juicio la investigación del arquitecto es "una invención que se plantea como tarea la organización y la construcción de un objetivo práctico concreto" 9 .

No parece extraño que la administración como actividad se convirtiera en la gran demandante de espacio para su desarrollo. Siendo la 
' Ver la voz: Trabajo duro en Saul John R. Diccionario del que duda: un diccionario de agresivo sentido común. Ed. Gránica, Buenos Aires,

${ }^{2}$ William, Raymond. Palabras clave un vocabulario de la cultura y la sociedad. Ed. Nueva Visión, Buenos Aires, 2000
${ }^{3}$ Banham, Reyner. La Atlántida de hormigón. Ed. Nerea, Madrid,

4 Ver Cohen, Jean-Louis. El tío Sam en el país de los soviets: el tiempo de las vanguardias. En Strigalev, A. A.; Cooke C. y Cohen, Jean-Louis. Constructivismo
${ }^{\mathbf{5}}$ Taylor, Frederick Winslow. The principles of scientific management. Ed. Harper \& B

- Para Taylor la administración científica se basaba en el estudio de tiempos y estándares de producción estandarización de herramientas e instrumentos, la verificación de excepciones o desvíos de patrones normales; la utilización de instrumentos destinados a economizar tiempo, las guías de instrucciones de servicio; la idea de tarea asociada a incentivos de producción ción input-output de materias primas and social change. En Art Journal Vol. métodos del pensamiento arquitede productos y el diseño de la rutina $\quad 43, \mathrm{~N}^{\circ} 2($ summer, 1983) pp. 32-147. tónico. En Hereu, Pere; Montaner, e trabajo. El punto más original fue

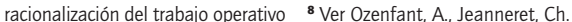
E. Après le cubisme (1918). En
Ozenfant, Le Corbusier. Acerca del pursimo. Escritos 1918-1926. Ed. El Croquis, Madrid, 1994. p. 2 osep María y Oliveras, Jordi. Textos de arquitectura de la modernidad. $\mathrm{Ed}$. Nerea, Madrid, 1994. p. 264.

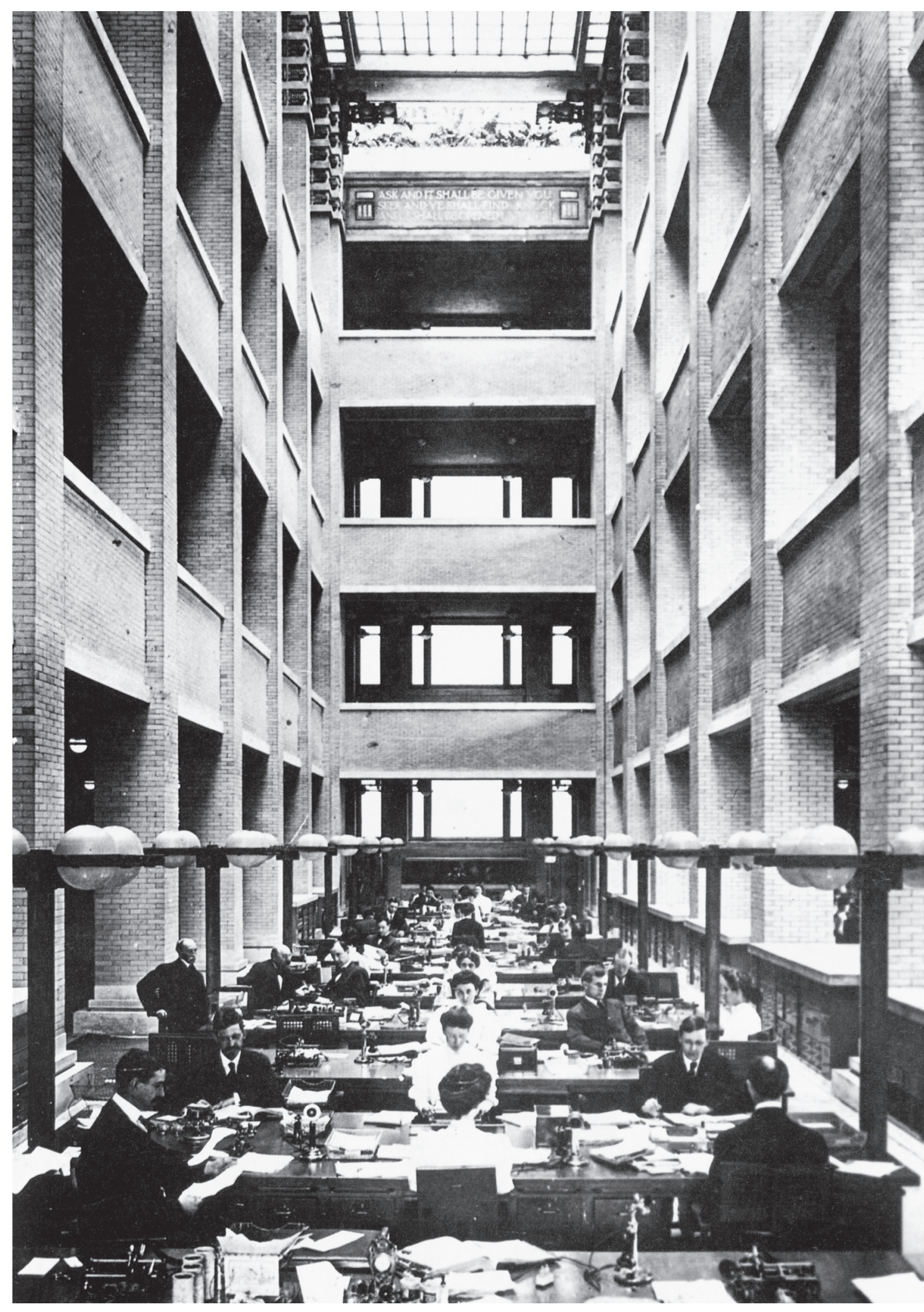


tarea administrativa, la que estudiaba los tiempos, planeaba las tareas, controlaba el input-output de la fábrica, establecía estándares y rutinas de trabajo, necesitaba entonces de una definición arquitectónica.

Las preocupaciones de la Arquitectura Moderna tanto en torno a la fábrica como a la oficina, estuvieron centradas básicamente en la organización de la planta, como espacio de la secuencia de tareas, y en la iluminación, como requerimiento básico del ambiente laboral. Fue en efecto la fábrica la que le prestó su método de secuencia ${ }^{10}$ al edificio de oficinas.

El programa de oficinas que hoy parece inocuo y repetido se canonizó como problema de arquitectura en los primeros años del s. XX. Hay dos casos en que método, pensamiento y creación parecen situarlos como claros paradigmas de la consideración del trabajo de oficina en el proyecto de arquitectura: el edificio Larkin de Wright y el proyecto de un rascacielos para la Friedrichstrasse de Mies van der Rohe.

El primero fue construido por Frank Lloyd Wright en 1904, como sede administrativa de la compañía Larkin. como parte de la planta productora de jabones que la empresa tenía en Buffalo. Como ha sostenido Frampton fue el primer intento de Wright de convertir un espacio de trabajo en un dominio sacramental ${ }^{11}$. Luego Wright lo desarrollaría aún más en el espléndido salón de la administración de la Johnson \& Son en Racine, Wisconsin en 1936-39.

El edificio Larkin era un gran edificio de planta rectangular, cerrado hacia fuera por muros de ladrillo, con cuatro macizas torres en las esquinas. El interior era básicamente una nave central de la totalidad de la altura, con iluminación cenital y tres pisos que la circundaban. La organización de los puestos de trabajo se daba en un plano abierto, tanto en el espacio central en el piso bajo, como en las alas laterales superiores. Este plano abierto, para la disposición de los escritorios, es decir para la organización de un estándar administrativo, es germen explícito de lo que hoy denominamos planta libre. Además, la liberación del espacio para el orden de los puestos de trabajo, establecía en principio un orden aparentemente neutro que venía reforzado por el retiro a los planos laterales de toda la utilería administrativa: los muebles de archivo modulares quedaban incorporados en los muros laterales, bajo las altas ventanas lineales. La luz diáfana proveniente del centro y de las ventanas laterales completaba la homogenización del ámbito de trabajo.

El Larkin fue pionero también en el acondicionamiento climático. Wright instaló un sistema de aireación por medio de grandes conductos para mover y calentar el aire -hasta la incorporación de aire frío en



02 
10 Bellucci, Alberto. Los envases de la sociedad terciaria. En Summa

N¹09 (febrero, 1977) p. 23.

" Frampton, Kenneth. 1903 Frank

Lloyd Wright: Larkin Building

Buffalo, New York, U.S.A. En GA

Modern Architecture. p. 126.

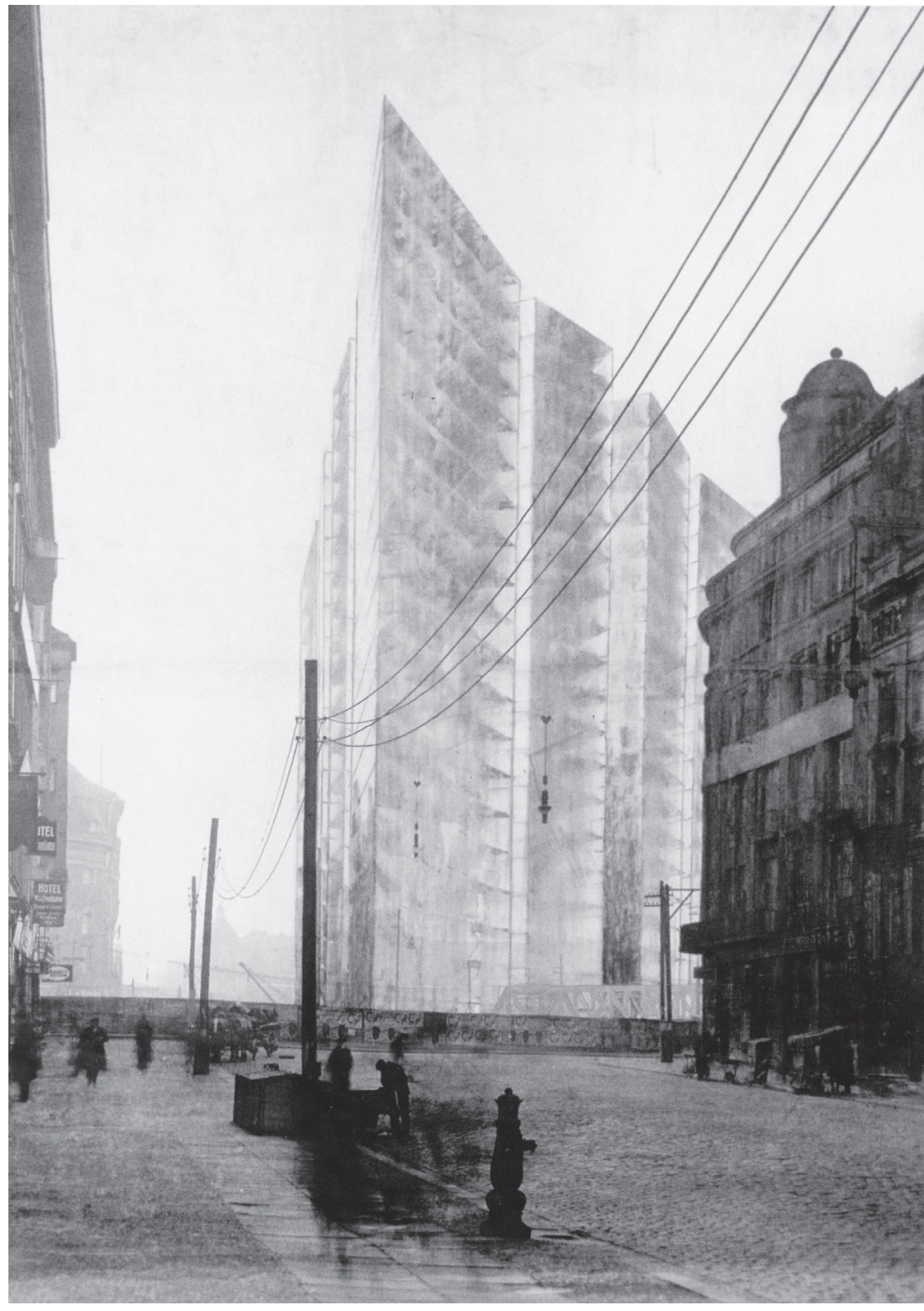

04

02 Salón de la administración de la Johnson \& Son en Racine,

Estados Unidos. Frank Lloyd Wright, 1936-39.

03 Planta piso tipo, proyecto rascacielos Friedrichstrasse en Berlín, Mies van der Rohe, 1921.

04 Perspectiva desde el norte, proyecto rascacielos Friedrichstrasse en Berlín, Mies van der Rohe, 1921. 




05
1909- con tomas de aire que estaban en la parte superior de las torres. Como anota Banham, el principal mérito radicó en que la propuesta de la amplia nave y la disposición de los servicios para lograr la ventilación, dio la forma magistral al exterior ${ }^{12}$.

En palabras del propio Wright era: "Una expresión sencilla de una llanura, un tipo utilitario con muros enteros en ladrillo y unas simples líneas superiores en piedra"13, a la vez que era "un ensayo sobre la tercera dimensión, un conservador reconocimiento de los elementos de la máquina en la vida moderna"14. En cambio, según Lewis Mumford: "Las oficinas -en su masa brutal- exhiben la inquebrantable intensidad de la vida moderna en este tiempo" ${ }^{15}$, entendiendo que la clave de resolución arquitectónica estaba en la presencia del edificio. Sin embargo, la correspondencia entre el tipo utilitario y la idea del ensayo maquinista parece haber sido la clave más importante y definitiva. Y ello no radica en un tipo particular de expresión en relación con los nuevos tiempos o con algún estilo maquinista, sino más bien en la disposición y alineación de los trabajadores en un ambiente controlado climáticamente.

El segundo fue el proyecto para el concurso de un rascacielos en la Friedrichstrasse, en Berlín en 1921. Fue la oportunidad en que Mies van der Rohe desarrolló su aporte inicial a la evolución del tipo del edificio de oficinas. Bajo el seudónimo Panal, -una referencia indirecta pero frecuente al trabajo- ${ }^{-16}$ Mies presentó un proyecto que ocupaba completamente el sitio de referencia y desconocía en parte las bases del concurso. Era un prisma de base triangular, subdividido en tres cuerpos, de 20 pisos de altura -unos $80 \mathrm{~m}$ - enteramente revestido en cristal, con facetados y algunos ángulos. En el centro geométrico disponía el núcleo de circulaciones que estaba dividido en tres partes -en relación con cada una de las partes del edificio-, cada una con seis ascensores, un sistema de escaleras, un sector de posibles recintos sanitarios.

Diría Mies hacia 1922: "El sitio era triangular, traté de usarlo completo, la profundidad del sitio me obligó a agrietar los frentes, para que el centro interior recibiera $l u z^{\prime 17}$. Lo que Mies aportaba con esta definición del proyecto era una consideración básica en la configuración del edificio de oficinas: la relación entre el perímetro y el núcleo. Claramente la operación de proyecto consistente en penetrar el volumen con estas grietas para conseguir iluminar la totalidad de la planta con luz natural, es dependiente de esa relación. En una figura geométrica perfecta, a mayor perímetro le corresponde mayor superficie y por tanto mayor distancia al centro geométrico. La extensión del perímetro, por medio de la inserción de vacío de tres figuras con ángulos, despliega el problema en tres partes y asegura una menor distancia al centro geométrico de las nuevas partes en que se ha dividido la planta. De este modo, la máxima iluminación se aseguraba no sólo por la mayor altura de piso a cielorraso, sino por las entradas que cortaban el volumen, por los pliegues de la piel y definitivamente por la fachada completamente cristalina.

En relación con un proyecto posterior, el edificio de oficinas en hormigón armado, de 1923, Mies sostuvo que: "El edificio de oficinas es una casa del trabajo, de la organización, de la claridad, de la economía. Grandes salas de trabajo luminosas, diáfanas, sin obstáculos para la vista, sin subdivisiones, articuladas únicamente a semejanza de la propia organización de la empresa. Máximo efecto con el menor empleo de medios"18. Inmediatamente más abajo, incorporaba un nuevo criterio en la relación antes establecida: "La distribución más adecuada de los puestos de trabajo determinó la profundidad del edificio"19. Por tanto, determinar la profundidad de la planta es al mismo tiempo determinar el perímetro y la figura de la planta.

Pero fue también Wright quien había ya racionalizado los componentes de la planta del edificio de oficinas, en el proyecto de un rascacielos en hormigón armado que desarrolló para San Francisco en 1912. Propuso 
12 Ver Banham, Reyner. La arquitectura del entorno bien climatizado. Ed. Infinito, Buenos Aires, 1975, pp. 90-97.

${ }^{13}$ Wright, Frank Lloyd. In the cause of architecture. First paper. March 1908. En Frank Lloyd Wright. The complete 1925 Wendingen Series. Dover Publ-
${ }^{14}$ Wright, Frank Lloyd. In the cause of architecture. Third dimension. May 1914. En İdem. p 56.

15 Berlage, H.P. Frank Lloyd Wright. En İdem. p 84

${ }^{16}$ Si bien algunos autores dan a conocer una posible referencia a Sullivan, que había usado el término en su ensayo The tall building artistically considered en 1896, la referencia a la colmena como sociedad trabaJera era vigente Ver Riley Ten jadora exa vigente. Ver Riley, Terence y Bergdoll, Barry. Mies in Berlin. Ed. MOMA, Nueva York, 2002

"Ver Riley, Terence y Bergdoll, Barry. Mies in Berlin. Ed. MOMA, Nueva York, 2002. p. 180.
${ }^{18}$ Mies van der Rohe. Edificio de oficinas (1923). En Neumeyer, Fritz. Mies van der Rohe la palabra sin artificio. Ed. El Croquis, Madrid, 1995. p 363.

19 Ídem, p. 364.

${ }^{20}$ Belluci, Alberto. Op Cit. p. 27.

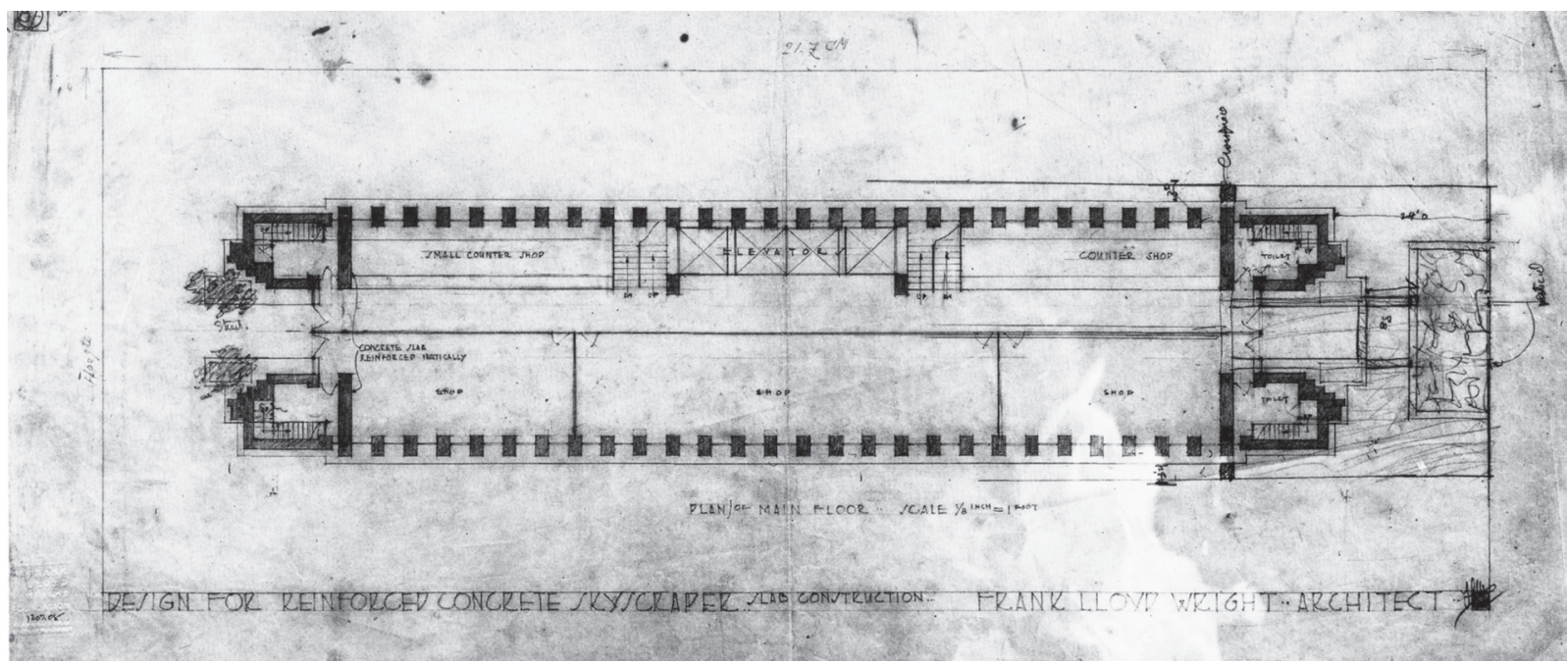

06

entonces un esquema de planta lineal y bipartita, con el núcleo de circulaciones en posición lateral. La estructura portante recaía en la fachada, en una serie ritmada de columnas que dejaban entre sí líneas acristaladas. La secuencia de líneas verticales marcaba una tensión ascendente y una visera superior otorgaba una sensación de liviandad a un edificio que era esencialmente bastante pesado. Internamente, las posibilidades de organización y subdivisión estaban dadas por la relación entre la circulación al interior de la planta y la correspondencia entre subdivisión y columnas de la fachada. La planta, más larga que ancha, permitía una organización del espacio de trabajo, modulado por las líneas de las ventanas entre columnas que daban luz natural. Nuevamente, la relación entre planta e iluminación natural estaba presente, pero esta vez, con un criterio diferente que establecía además condiciones diferenciales al espacio.

El espacio inmediato de trabajo fue ensayado por Wright en el edificio Larkin y posteriormente fue más desarrollado en la Johnson Wax. Su concepción estaba en las líneas del edificio, como una obra de arte total. En principio, suponía una continuidad estilística entre edificio y puesto de trabajo, pero iba más allá, en la consecución del diseño total del entorno.

En el Larkin se asumía que la repetición en extensión de escritorios debería tener un tratamiento que mediara la situación alienante del lugar de trabajo entre tantos otros trabajadores con el total del edificio; es decir asumir condiciones del edificio en el puesto de trabajo para intentar anular la distancia entre el operario y la gran nave. Sus pretensiones de diseño total llegaban desde el mobiliario hasta los teléfonos, cuestión que la empresa abortaría tempranamente.

Si bien la pretensión wrightiana es muy de época y se corresponde a una Gesamtkunstwerk -obra de arte total-, el diseño del puesto de trabajo en relación con el edificio surgió frente al anonimato que impone el espacio de las oficinas, aparentemente desprovisto de carácter, y al desarraigo que propone para el empleado ${ }^{20}$.

La arquitectura ha enfrentado algunas opciones. Una de ellas, parece estar definitivamente asociada a una escala inmediata al cuerpo. La oficina mínima, que se ubica disciplinariamente entre la arquitectura y el diseño, como es ampliamente conocido, ha llevado a innumerables propuestas en el campo de diseño de interiores y mobiliario, llegando hasta dispositivos diseñados para contener en un mínimo espacio la mayor cantidad de útiles de oficina. Ya con autonomía del edificio, el módulo de trabajo con diseño total integrado, permitió una libertad de organización de la planta, de acuerdo con los cambios que el organigrama de la empresa podría asumir.

Para Le Corbusier, el concepto de planta libre se equipara con la no correspondencia entre estructura portante y subdivisión programática; lo que habitualmente llamamos planta libre en el edificio de oficinas, se corresponde también con una planta neutra, es decir liberada de condiciones programáticas específicas y al mismo tiempo capaz de permitir una amplia variación en el trazado de los alimentadores de los distintos sistemas de acondicionamiento, básicamente lumínicos, eléctricos y de aire acondicionado. A ello contribuyó el cielorraso suspendido, pero también una nueva formulación de la estación de trabajo y un método de proyecto por módulos, que permitieron máxima flexibilidad para asumir distintas condiciones de formalización del espacio de trabajo. Las técnicas del proyecto del edificio de oficinas estarán dominadas desde ese tiempo por las relaciones entre iluminación, núcleo de circulaciones, disposición posible de mobiliarios y superficie disponible de planta neutra. 
${ }^{21}$ Ginzburg, Moisei. Op. Cit. p. 267.

${ }^{22}$ Ver Tafuri, Manfedo y Dal

Co, Francesco. Arquitectura

Co, Francesco. Arquitectura
contemporánea. Ed. Aguilar, Madrid,

contemporánea.

Comentario obras y proyectos / Los casos incluidos en esta revista, que motivan este comentario transversal, nos muestran una gama bastante amplia de resultados sobre algunos de los tópicos expuestos. En todo ellos se supone que hay o habrá algún número de personas ocupadas en sus trabajos; mayoritariamente son oficinas, y con algunas variaciones, proponen distintas lecturas de las técnicas más habituales para la configuración de los lugares de trabajo. Un primer grupo, conformado por cinco casos de edificios de nueva construcción, muestra las posibles variaciones en la determinación de la planta, en relación con la iluminación natural, la superficie disponible y el perímetro. Si bien en todos los casos las propuestas surgen de las particulares condiciones del sitio y de las normativas vigentes, las memorias destacan con claras referencias el problema de la configuración de la planta en relación al núcleo y a la iluminación natural.

El Abu Dhabi Commercial Bank, aún cuando pudiera relacionarse con el rascacielos de San Francisco de Wright, incluso por su visera, presenta una planta tipo singular, de proporciones similares al edificio Larkin. Al ubicar los dos núcleos de circulaciones en los lados, libera al centro la superficie útil para la disposición flexible de los puestos de trabajo. La opción propone una jerarquía en la iluminación sólo por los otros dos lados del perímetro. Sin embargo, la utilización de planos transparentes en los núcleos de ascensores completa las posibilidades de recepción de luz natural al interior, como un dispositivo de proyecto superador de las formas y relaciones ya tradicionales.

El edificio Consorcio en Concepción muestra una opción por ubicar el núcleo en posición lateral, en relación con el medianero, que se configura como un volumen que define la relación con el mismo. Las plantas neutras, con el máximo de superficie libre con orientación norte, se manifiestan como otro volumen vidriado que recibirá el tamiz de luz que proponen los perfiles de madera laminada y la vegetación.

En el caso del edificio Santa María se manifiesta la intención de alejarse de la configuración del volumen compacto con planta rectangular que caracteriza habitualmente al edificio de oficinas. La ubicación central del núcleo de circulaciones y su exposición al frente del edificio permiten configurar lo que en apariencia son dos volúmenes acentuados por los diferentes tratamientos de las fachadas.

El edificio Costanera en Puerto Montt muestra, de manera convincente, la relación entre planta neutra, iluminación y estructura portante. Como en el rascacielos de Wright, a la liberación de la planta le corresponde la presencia estructural en la fachada, y por tanto su condición de regularidad y alternancia para permitir la presencia de la luz al interior. La decisión de configurar una planta rectangular en el medio del sitio es acompañada por la posición simétrica y central del núcleo de circulaciones. Los planos de iluminación son directos en el frente y el contrafrente, y articulados por quiebravistas en los costados laterales.

El edificio CCU contrasta claramente con sus vecinos en el área, y no es tan solo por su relación urbana, siendo el único que ha sido generoso con el lugar, sino que también por poner en juego de manera renovada las temáticas arquitectónicas del edificio de oficinas. Se trata de una planta racionalmente establecida en una relación entre longitud y ancho, y la posición del núcleo de circulaciones verticales, en relación con el perímetro, para la obtención de mayor iluminación natural. La disposición de una planta más alargada tiene las ventajas que se señalaron en el edificio de Wright y que se verifica en los pisos altos, una planta similar, con el núcleo en un lateral. Asimismo, la fachada con rol estructural en los cabezales anula la superficie plana del reflejo.

El segundo grupo está conformado por intervenciones en el interior de edificios existentes, por lo que ponen el acento en la disposición de los módulos de trabajo y la relación con el perímetro disponible. En una excepción, las oficinas de la Dirección del Zoológico, probablemente por su situación, proponen otros temas y problemas.

En las oficinas Turner, la intervención en el antiguo edificio propone la creación de un jardín interior, en correspondencia con el lucernario existente, para generar una doble entrada de luz. El recurso Larkin es evidente y a la vez superado por la ubicación de las oficinas más cerradas hacia el interior de la planta, envolviéndolas en vidrio rojo, y dejando los puestos de trabajo hacia los bordes.

En las oficinas Philippi, Yrarrázaval, Pulido \& Brunner, las características distributivas vienen fuertemente dictadas por la proporción de la planta y la ubicación del núcleo. La utilización de un dispositivo espacial como son los pilares que ritman el espacio y dejan pasar la luz, permite la disposición de las oficinas sobre el perímetro, sin ahogar el centro. A la vez, los vacíos en las losas en los extremos refuerzan la condición de iluminación, aproximando otras dimensiones espaciales.

La intervención en las oficinas BBDO, la opción es bastante radical. Si en el edificio Larkin, el muro-mueble-archivo reforzaba la neutralidad de la planta, en este caso, la relación entre superficie de oficina y superficie de archivo es determinante en el proyecto. Los puestos de trabajo están en relación con la luz plena. El muro-mueble es, en definitiva, el proyecto de arquitectura.

El tercer grupo radicaliza la opción del espacio de trabajo como el módulo mínimo y aislado, lo que pone en evidencia muchas otras relaciones. La pequeña escala de aproximación aparece representada en este contexto por dos obras: la consulta Cubo y el Cabanon de Le Corbusier. Ambas emplazan una relación distinta: en aislamiento, construyen un mundo íntimo. En la consulta Cubo, para el trabajo domiciliario pero aislado de la casa, construyendo un abstracto. En el Cabanon, para el tiempo libre, o para una modalidad más libre del trabajo (mi oficina lo llama en la carta). La condición de mínimo propone un concepto de eficiencia que puede asumirse como la neutralidad total o como el establecimiento de dispositivos arquitectónicos ajustados a fines precisos.

La práctica del proyecto se enfrenta a los componentes clave que han tenido vigencia durante el s. XX: la organización de la planta, la luz y, consecuentemente, la definición de la fachada, la concepción de un clima artificial -aire acondicionado-, la relación entre mobiliario de archivo y espacio, y la relación entre mobiliario y espacio íntimo. Obviamente, no quedan fuera otros intereses, como la relación con el lugar u opciones más particulares o personales sobre arquitectura. Pero en edificios que son dispositivos para orientar las prácticas en relación con la eficiencia el método de creación funcional todavía sigue pesando. Sobre todo porque la práctica arquitectónica en este tema tiende a la eliminación de todo lo superfluo. Para Ginzburg, del método de la creación funcional se derivaba necesariamente un ascetismo, que obligaba a "rechazar lo inesencial y a buscar la expresividad artística en lo más importante y necesario" 21 . Porque, como dijo Tafuri en referencia a Loos: "La realidad de la división del trabajo contiene en sí la superación de todo acto superfluo" ${ }^{22}$. ARQ 